\title{
Iniciativas digitales y tecnológicas para la educación
}

\section{doi: $10.52749 /$ fh.v2i4.4}

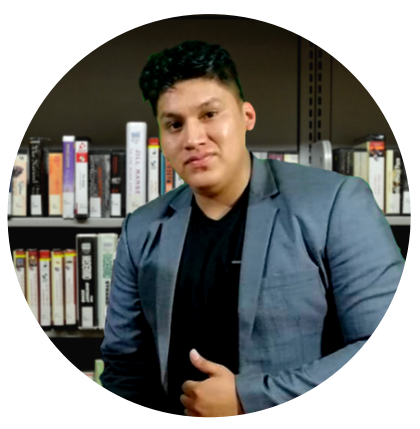

\section{KENNER MORI CASTRO}

\section{https://orcid.org/0000-0002-4749-9166}

CEO y cofundador de Innovacit, fundador de la organización social Cultura Steam, divulgador de ciencia, tecnología y educación. Miembro del Consejo Regional de Ciencia, tecnología, emprendimiento e innovación de Huánuco (CORCITEI). Miembro Investigador del Instituto de Estudios Transhumanistas (IET). Formó parte del Consejo Regional de la Juventud - Huánuco. Lic. en Sociología por la Universidad Nacional Hermilio Valdizán, egresado de la Maestría en Gestión Pública para el Desarrollo Social y estudiante de Ingeniería de Sistemas computacionales.

kenner.yesser@gmail.com

@kennermori19

Resumen. Las revoluciones industriales se han caracterizado por mantener un auge y marcar una tendencia a lo largo del proceso histórico-cultural de la humanidad. Esto se debe a que el avance científico y tecnológico es una realidad innegable que contribuye con el desarrollo humano. Como resultado, los avances tecnológicos han traído consigo un marcado progreso exponencial, tanto en conocimiento, como en problemáticas de tipo social y económico. Esto se debe a que los estados y las revoluciones involucran ciertas manifestaciones previas, tales como: El crecimiento asimétrico de los recursos, la diferenciación en todos los estratos y la generación de un sistema nuevo dentro del actual. Razón por la cual, una formación científica y ética requiere una cultura consolidada bajo una perspectiva humanista. Para ello, el presente proyecto consiste en una investigación teórica, enmarcada en los hitos de la revisión narrativa-conceptual, acerca de aquel constructo definido como Cuarta revolución industrial. Asimismo, se han de examinar los principales impactos de este paradigma emergente en las dimensiones biologicas, psicológicas, sociales y contextuales de la sociedad.

Palabras clave: neurotecnologías, neurohackers, transhumanismo

Vivimos en la "era del internet", de la "IV Revolución Industrial", donde el acceso a la educación y desarrollo de nuevas habilidades suele ser más fácil para algunos y difícil o imposible para otros. Debemos considerar que la educación hoy en día se ha convertido en una carrera contra el reloj por no dejar a nadie atrás, por el desarrollo de la automatización y la adaptabilidad a nuevos contextos que día a día cambian y nos exigen estar más preparados. Nuestro sistema educativo es heredero de un contexto social pasado que no usa ni aplica las tecnologías presentes eficazmente y niega un panorama social y económico muy distinto y cambiante en un futuro próximo.

"En el caso peruano y en el de muchos países de la región latinoamericana, nuestros sistemas educativos se edifican sobre muros débiles. Escuelas en zonas rurales, e incluso urbanas, sin acceso a internet o medios tecnológicos, docentes poco capacitados, y la corrupción a todo nivel, son algunas de sus características". (Mori Castro, 2020, p.19)
¿Qué iniciativas existen para mejorar el acceso a la educación y aprendizaje de nuevas tecnologías?

Antes de citar algunas iniciativa es sustancial reconocer el papel del docente en todo este proceso para la mejora socioeducativa, debido a que el educador tiene que ser un animador social en el sentido de que crea situaciones de aprendizaje, impulsa la realización de esas actividades, las pone en marcha e incita a que los alumnos las desarrollen, las lleven adelante, y les ayuda y orienta en las dificultades (Delval, 2013). Si bien, la docencia es una de las actividades que no van a ser reemplazadas en el mediano plazo, existen iniciativas que pueden ser el soporte y la llave para el cambio educativo que necesitamos.

La IV revolución industrial que estamos viviendo, el surgimiento de nuevas tecnologías y el incremento en la digitalización en las empresas nos exige que mejoremos ipso facto la forma como enseñamos y las habilidades que forjamos para el mercado y el futuro. La programación, ciencia de datos y muchas ingenierías en tecnologías son algunos de los trabajos más demandados en el siglo 
$\mathrm{XXI}$, puesto que, este lenguaje digital potencia la creatividad, desarrolla el pensamiento crítico, otorga habilidades para la mejora de resolución de problemas reales y sobre todo tiene una demanda laboral muy grande.

De acuerdo al estudio publicado por el Foro Económico Mundial (2018), denominado "El Futuro del Trabajo 2018", en el 2022 se calcula la creación de 133 millones de empleos a nivel mundial, no obstante, estos empleos se van a centrar en sectores de la revolución digital o Cuarta Revolución Industrial.

Aprender sobre código ya no es un área exclusiva de las universidades; muchas escuelas de educación básica regular están iniciando a enseñar este nuevo idioma digital acompañado de la robótica y otras habilidades que acompañan el desenvolvimiento de los educandos en la sociedad actual.

Nombremos algunas de las iniciativas que plantean democratizar el acceso a la educación y el aprendizaje de la programación, robótica y nuevos conocimientos tecnológicos.

1. La primera iniciativa sin fines de lucro tiene como nombre "Code.org." Es dirigido por el inversor tecnológico y director ejecutivo Hadi y Ali Partovi, quien es graduado de Harvard con una maestría en Ciencias de la Computación. Este es uno de los proyectos sin fines de lucro más grandes y sorprendentes en cuanto a la enseñanza sobre las bases de las ciencias de la computación mediante la programación en bloque y código para distintos juegos como Angry Birds, Minecraft, Code Combat, Code Monkey y muchos más que son conocidos por cientos de millones de estudiantes en todo el mundo que aprenden de manera divertida. Además, Hadi promueve "The hour of code" (la hora del código), que es un movimiento a nivel mundial para celebrar las ciencias de la computación en colegios, barrios, distritos y demás lugares mediante la plataforma online o algunas actividades offline para zonas que no cuenten con internet.

En la plataforma puedes aprender sobre las bases de la programación web, programación de apps y de distintos lenguajes de programación como Python, CSS, JavaScript etc. En muchos de los videos para promocionar la "Hora del código" y con la frase "Todo el mundo puede aprender a programar", encontramos al ex presidente Barack Obama, empresarios como Mark Zuckerberg, Bill Gates y muchos otros CEOs de empresas en tecnología, deportistas y cantantes que animan a los estudian- tes a usar Code.org y ser parte de esta iniciativa.

Por último, este proyecto ha sido adoptado por muchas instituciones educativas en el Perú y otros países, pero muchos solo los tienen como referencias externas al desarrollo curricular y no existe un plan dirigido exclusivamente para la enseñanza de los recursos de Code.org. u otras plataformas.

2. La segunda iniciativa se llama "Tinkercad",el cual es un software gratuito, que fue creado en 2011 por el ex ingeniero de google, Kai Backman y Mikko Mononen (cofundador), pero luego, en el 2013 fue adquirido por Autodesk. Esta plataforma nos permite programar, simular y ensamblar circuitos electrónicos, así como piezas, objetos en 3D y diseño con código, todo esto en línea.

Un recurso muy interesante para las instituciones de educación básica, superior y cualquier persona interesada en estos temas. Si muchas instituciones educativas contaran con impresoras 3D, su uso iría incluso más allá, por ejemplo, a la creación de sus propios robots, debido a que podemos diseñar las piezas robóticas en 3D, imprimirlas y armarlas. Asimismo, la enseñanza de la electrónica es más fácil, puesto que, mediante prueba y error evitamos malograr componentes físicos y gastos innecesarios a los alumnos.

3. Otra iniciativa, en este caso exclusivamente para la enseñanza de programación y creación de Apps es "MIT App Inventor." Este proyecto gratuito, como se menciona en su plataforma, busca democratizar el desarrollo de software al empoderar a todas las personas, especialmente a los jóvenes, para pasar del consumo de tecnología a la creación de tecnología.

Aquí los educandos pueden crear aplicaciones que son funcionales para los teléfonos inteligentes (Smartphones) y tabletas de Android o iOS. Su programación está basada en bloques y esto facilita la creación de muchas aplicaciones complejas para solucionar problemas reales y de brechas existentes. Todo es cuestión de una conexión a internet, creatividad y aprendizaje constante ya sea individual o en grupo.

4. Imagina, programa y comparte. Uno de los entornos de programación gratis más interesantes para la creación de sus propios juegos en línea o historias es "Scratch". Creado por un grupo de investigadores del Instituto de Tecnología de Massachusetts (MIT) esta plataforma gratuita aprovecha los avances en el diseño de interfaces para hacer que la programación sea atractiva y accesible para las personas en especial para niños y 
adolescentes que por primera vez van a conocer el mundo de la programación.

Lo más resaltante de esto es que podemos aprovecharla para la enseñanza en centros educativos donde los niños y adolescentes harán uso de la imaginación, creatividad y bloques de código para crear su propio juego que puede transmitir una historia real, ficticia, regional o histórica y publicarlo en línea para que millones de otros usuarios lo puedan jugar. El educando explora la tecnología creando y siendo partícipe de ella.

5. Para sumergirnos a la programación en blobloque y observar cómo se mueve un robot digital con instrucciones del usuario, "VEXcode VR" viene a ser una plataforma muy útil y sobre todo gratuita. Los que ya hemos compartido conocimiento con niños y niñas sobre programación y robótica entendemos que gran parte de su aprendizaje va de la mano con lo que ellos experimentan, sienten y los obstáculos que vencen de manera individual y grupal para el desarrollo de una actividad. Es decir, el niño aprende equivocándose y poniéndose nuevos retos en el juego.

En esta plataforma podemos hacer que los estudiantes muevan el robot mediante el uso del Block Coding o Python para escribir código. Incluso esta plataforma tiene un Vex Robotics Off-Road Truck, que es un vehículo controlado a distancia que se ensambla y se puede controlar con la App de VEX PILOT.

6. El internet es una de las creaciones e instrumentos más valiosos en el siglo XXI y es necesario aprovechar al máximo sus recursos. El aprendizaje en línea es un gran aliado para docentes, estudiantes y una de las organizaciones sin fines de lucro que tiene un gran impacto en este tema es "Khan Academy". El creador de esta grandiosa plataforma es el educador Salman Amin Khan.

Esta iniciativa es una plataforma web donde aprendemos mediante videos de manera ordenada y por etapas sobre distintos temas como electrónica, ciencias de la computación, biología, finanzas, economía, matemática, cálculo, álgebra, química y otras áreas con ejercicios prácticos e incluso evaluaciones. Es una de las primeras escuelas virtuales en el mundo que sirve como recurso educativo para estudiantes de educación básica, superior y docentes.

Esta y otras iniciativas mencionadas incluso son buenos aliados de la estrategia didáctica del "Flipped Classroom" o "aula invertida", donde la enseñanza tradicional de docente a alumno en el aula cambia y muta hacia la participación activa del estudiante en clase previo conocimiento o área revisada.

\section{"La metodología del aula invertida propone que los estudiantes vean vídeos grabados, realicen lecturas, etc., previamente a la sesión, y que sea en la sesión con el docente donde se realice alguna tarea bajo su supervisión" (Rodríguez Herrero \& Ruiz Ambit, 2021, pág. 7).}

Al igual que la última iniciativa mencionada líneas arriba, existen otras con igual o mayor impacto en la educación. Los MOOCs (Massive Open Online Courses) que son cursos masivos en línea vienen a ser una modalidad de formación online que es cursada por miles de estudiantes en internet. En los últimos 10 años hubo gran relevancia en la mejora académica de muchas personas y la democratización al acceso de cursos de universidades del extranjero como Harvard, MIT, Stanford, Berkeley, Boston University, Universidad de Pensilvania, Universidad autónoma de Mexico entre muchas otras de distintos países. En esta oportunidad solo mencionaré algunas de las más influyentes, como son, edX.org, Coursera.org, MiriadaX, Future Learn, UniMOOC, Tutellus etc. Por otro lado, otras universidades ya empiezan a crear sus propios cursos para ofrecerlos en alguna de estas plataformas o crear la suya, eso sin duda permite el acceso de más estudiantes que acceden al conocimiento y experiencia de docentes y profesionales no solo de la región sino a nivel nacional e internacional.

En estas plataformas encontramos distintos programas y cursos que van desde humanidades, literatura, diseño, idiomas, finanzas o ingenierías en áreas como el desarrollo de software, programación, ciencias de la computación, robótica, inteligencia artificial, ciencia de datos, electrónica entre otros.

Existen más profesionales y emprendedores que cuentan con iniciativas como estas, que forman parte de comunidades digitales o son actores sociales para la transformación educativa. Incluso es necesario explorar nuevas tecnologías como el uso de video juegos para enseñar distintas áreas escolares, la realidad virtual, realidad aumentada, la inteligencia artificial o pensar en cómo utilizar las tecnologías futuras como el Metaverso de Zuckerberg para apoyar la educación en todo nivel.

Las 6 iniciativas descritas en este artículo son solo algunas de muchas, ya sean gratuitas o de paga, lo más importante es que este ecosistema de plataformas en línea para mejorar las habilidades 
educativas y tecnológicas democratizan el acceso a la educación y hacen posible maximizar el conocimiento en el área requerida o sumergir a los niños en nuevas experiencias con tan solo contar con un ordenador e internet. Otras plataformas para adquirir conocimiento en distintas áreas son: Code Academy, Solo Learn, Plural Sight, Código facilito, Udemy, Platzi, Code Avengers, EDteam, Aprende.org y la lista continúa aún más.

Para culminar este recorrido, es importante mencionar que, aunque me considero un tecnooptimista no debemos caer en el solucionismo tecnológico, por el contrario, es necesario analizar el contexto donde usar los instrumentos de educación tecnológica y hasta qué punto puede ser de gran ayuda o complemento. Aún permanece la tarea ardua de aprender y adoptar mucho de lo planteado en este artículo, además de utilizar otros enfoques de enseñanzas como el enfoque "STEAM", planear un nuevo rumbo educativo y ejecutar políticas públicas que vayan más allá de papeles y simples informes y se plasme en la realidad con cambios trascendentales en los niños, jóvenes y demás profesionales, sin importar en contexto geográfico y cultural.

\section{Referencias}

Delval , J. (2013). La escuela para el siglo XXI. Sinéctica - Revista electrónica de educación, 14. http://www.scielo.org.mx/scielo.php? script=sci_arttext\&pid=S1665-109X2013000100004

Mori Castro, K. (2020). El reto educativo del siglo XXI: el enfoque STEAM en la Cuarta Revolución Industrial. Futuro Hoy, 1(1), 19. https://doi.org/10.5281/zenodo.4299184

Rodríguez Herrero, P., \& Ruiz Ambit, S. (12 de junio de 2021). ¿Qué es el aula invertida y por qué es la gran sorpresa de la educación durante la pandemia? BBC News. https://www.bbc.com/mundo/noticias-57559119

World Economic Forum. (2018). The Future of Jobs Report 2018. Ginebra-Suiza.

\section{Cómo citar este artículo:}

Mori, K. (2021). Iniciativas digitales y tecnológicas para la educación. Futuro Hoy, 2(4), 24-27. https://doi.org/10.52749/fh.v2i4.4

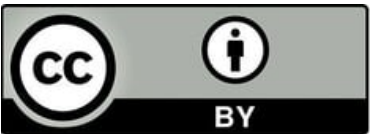

Esta obra está bajo licencia internacional Creative Commons 4.0 Reconocimiento 4.0. 